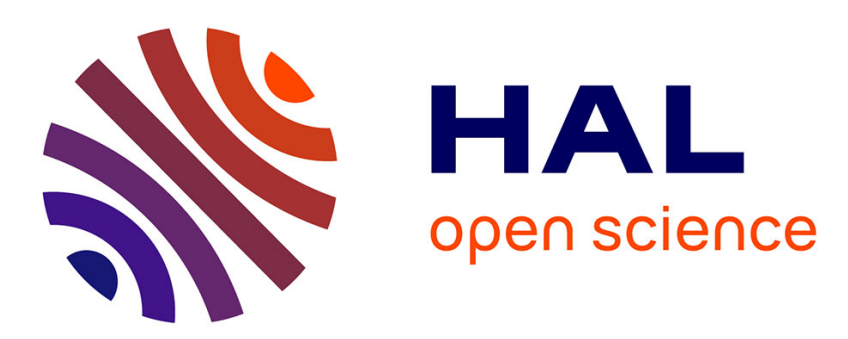

\title{
À propos de Dans les coulisses du genre: la fabrique de soi chez les Drag Kings, de Luca Greco
}

\author{
Béatrice Fracchiolla
}

\section{To cite this version:}

Béatrice Fracchiolla. À propos de Dans les coulisses du genre: la fabrique de soi chez les Drag Kings, de Luca Greco. Semen - Revue de sémio-linguistique des textes et discours, 2019, Discours de haine dissimulée, discours alternatifs et contre-discours, 47, pp.145-147. halshs-02490772

\section{HAL Id: halshs-02490772 \\ https://shs.hal.science/halshs-02490772}

Submitted on 2 Mar 2020

HAL is a multi-disciplinary open access archive for the deposit and dissemination of scientific research documents, whether they are published or not. The documents may come from teaching and research institutions in France or abroad, or from public or private research centers.
L'archive ouverte pluridisciplinaire HAL, est destinée au dépôt et à la diffusion de documents scientifiques de niveau recherche, publiés ou non, émanant des établissements d'enseignement et de recherche français ou étrangers, des laboratoires publics ou privés. 
Compte-Rendu par Béatrice Fracchiolla, Université de Lorraine, CREM EA3476

Luca Greco, 2018, Dans les coulisses du genre : la fabrique de soi chez les Drag Kings, Editions Lambert Lucas, Limoges.

Ce nouvel ouvrage de Luca Greco s'intéresse à retranscrire et expliquer ce que recouvrent les différents niveaux, contextes, performances possibles du genre. II se focalise ici sur la construction et à la présentation d'un soi " masculin ", à partir des résultats d'une enquête de terrain menée à Bruxelles entre 2008 et 2013 dans les ateliers Drag Kings. Véritable synthèse réalisée à l'appui d'une enquête de terrain, ce livre très documenté s'appuie sur un nombre de références et une vaste et complexe connaissance du domaine et réflexion sur le sujet, qui transparaît également dans l'écriture, à la fois dense et claire de Luca Greco.

L'auteur prend soin de préciser dès la première page d'introduction qu'il s'agit bien " d'un point de vue ", qui est celui " d'un ethnographe " "sociolinguiste " qui travaille " en études de genre " et pour qui le langage, dans tous ses aspects (voix, paroles, gestes, postures, vêtements, regards) est au centre de "l'accomplissement du genre ». Cette grille de lecture, propre à l'auteur, reste ainsi ouverte sur d'autres possibles. Ainsi, bien que son point de vue soit celui d'un linguiste, on voit d'emblée que le langage ne se réduit pas ici au seul verbe, mais s'enrichit d'autres perspectives " anthropologiques, sociologiques, théâtrales " (et plus encore), qui donnent une vision pluridimensionnelle du langage, comme quelque chose de dynamique, complexe, incarné par le corps et sa mise en scène autant que par les mots et leur mise en scène. L'ouvrage suggère que, de même qu'existe une syntaxe verbale, existe en même temps une syntaxe des corps, et de leurs décors. Autrement dit, un langage émerge, propre à l'affirmation des corps, avec ses propres caractéristiques en fonction du contexte. Une première partie propose une synthèse historique et épistémologique des recherches à propos et autour de ce sujet; elle s'appuie pour l'essentiel sur des recherches anglo-saxonnes, mais aussi sur des auteur·ice·s italien·ne·s. L'émergence des Drags Kings (DK) dans les années 90 est abordée dans la perspective d'une linéarité historique. Luca Greco s'attache à définir et surtout, retracer l'histoire et l'évolution de certains mots (camp, queer, butch, Drag king, etc.). Il parvient ainsi à rendre compte de toutes les problématiques associées à son terrain central (les DK), dont il fait une lecture toute en capillarité (sans jeux de mots), qui le mène à retracer l'histoire des notions et des liens qui les organisent entre elles, de ce que l'on pourrait appeler, du moins provisoirement, les " jeux de genre "- que ceux-ci correspondent à des choix d'expérimentations artistiques, personnelles (des performances), comme le Drag Kinging, ou à un parcours obligé lié à un vécu, un ressenti autre par rapport à l'assignation de genre lié au sexe de naissance. D'un point de vue théorique, I'histoire du travestissement, présenté d'abord comme fondé sur une approche externe est abordé ici à travers ses différences avec la notion de trouble, qui renvoie à une approche interne du rapport au genre, et reçoit des descriptions psychiatriques voire pathologiques : le Drag est présenté comme un jeu, une pratique - théâtrale aussi, qui ne se réduit pas au seul travestissement, mais cumule $d$ 'autres signes et mises en actes et en mouvement du travestissement (moustaches, marcher, parler, interagir, chanter...). L'historique proposé des Ftomale (Female to Male) jusqu'aux FtoUnknown (De femme à inconnu), montre la multiplication des catégories possibles dans la diversification des identités, en nuances. Une infinité d'incarnations possibles de l'autre genre est ainsi montré comme susceptible d'advenir, et à des degrés divers - dans la mesure ou l'autre genre ne saurait, lui non plus, recevoir une lecture ou une existence linéaire ou lisse. 
Dans le contexte de déconstruction et de libération d'un soi genré chez les $D K$, l'auteur explique comment le fait d'incarner plusieurs types de masculinités met à mal la dimension ontologique de la masculinité et du genre et montre son caractère construit, fictionnel, performatif (Connell 1995). Si l'ensemble de l'ouvrage vise à rendre compte de la dimension "catégorielle, collective, historique et politique des pratiques de construction et de présentation d'un soi masculin ", Luca Greco défend l'idée d'une "pluralité du soi » qu'il définit et illustre en s'appuyant sur l'idée de "rhizome " empruntée à Deleuze \& Gattary (1980). Le fil directeur qui conduit l'écriture de l'ouvrage est d'envisager la construction du genre comme un processus qui s'accomplit et se transforme au quotidien, et aussi tout au long de la vie. L'inscription temporelle, mais aussi spatiale et sociale sont au centre de ces expérimentations. Or, une pratique sociale ne peut se construire que de manière collective, intersubjective et interactionnelle, et au moyen de ressources langagières diverses. Ainsi, le langage est ici envisagé avant tout comme lieu de confrontation, d'actualisation des transformations et métamorphoses progressives du soi et d'édification des pratiques et des resignifications continues du travestissement.

Ce sont des pratiques politiques et artistiques dans lesquelles "le corps peut devenir un matériau plastique mais aussi la matière et l'objectif d'une transformation publique ". La question est ainsi plus de savoir comment les personnes accomplissent cette transformation que de savoir pourquoi il la font et la recherchent. Il s'agit d'étudier les modalités de leur faire, les processus (qui conduisent à une performance), pour sortir d'une vision psychologisante individuelle des pratiques quotidiennes.

Dans une seconde partie, l'auteur décrit plus en détail tout le long travail d'enquête ethnographique, mais aussi le parcours de vie et d'observation participative, nécessairement inscrit dans et le processus de récolte des entretiens. Sur le terrain, le rapport au théâtre et à la performance est immédiatement créé par la notion de changement d'espace mise en avant dans le fait d'un lieu séparé où les femmes (et parfois aussi des hommes) se maquillent - qui s'apparente à des coulisses théâtrales, pour filer la métaphore proposée par l'auteur - et le bar, où yels descendent ensuite, Drag Kings, prendre un verre et rencontrer un public. L'escalier qui descend de l'un à l'autre espace devient dans ce récit de recherche-action, le lieu de la transition, de la prise en charge du rôle.

Partie prenante de ces expérimentations identitaires, les choix d'écritures pronominales créatives proposées par les membres sur les listes de diffusion des ateliers $D K$ et explicités en fin d'ouvrage (yeal, yels, toustes, ceuses, celleux, nombreuxses), sont eux aussi adoptés à différentes reprises par l'auteur. Plus encore, au fur et à mesure de l'écriture et de la recherche, c'est l'idée des identités dégenrées, l'idée ( $d$ 'abord abstraite, mais rendue concrète par le récit de pratiques ancrées dans le réel) d'un possible non genre, et l'exploration des possibles identitaires au-delà du genre, qui sont proposées. Cela apparaît avec plus de force dans les dernières pages, lorsqu'il est question des Draqueens FtoF (incarnées donc, par des femmes, se déguisant en Dragqueens), des pratiques clowns, ou encore des explorations de figures masculines au-delà d'une quête du masculin qui passeraient par le viril (les vieux, les homos, les poilus, les nerds...). La conclusion de l'ouvrage demeure ainsi une porte ouverte sur les possibles, en particulier à travers les liens évoqués par l'auteur entre les pratiques $D K$ et d'autres pratiques (feldenkrais, body mindcentering approach, yoga) qui, comme il le dit, constituent toutes des voies menant à une " prise de conscience de son corps, de la façon dont il fonctionne et de la façon dont il est modelé par les habitudes, par le mouvement mais aussi des pratiques artistiques performatives qui mettent au centre le corps genré, son caractère plastique, ritualisé et politique. " (p. 151). Le livre de Luca Greco invite donc aussi à 
reconsidérer nos corps dans la perspective d'une harmonisation avec notre personne; la proposition d'une méditation sur l'alliage - et l'alliance - entre aspirations intérieures et théories de l'action; entre être et représentation. La perspective d'un intéressant voyage. 